Produto \& Produção, vol. 5, n. 3, p. 61-69, out. 2001

\title{
Captura-Recaptura: usando Estimadores Bayesianos para estimar o número total de defeitos distintos em inspeções de produtos
}

\author{
Sueli A. Mingoti, Ph.D. \\ Universidade Federal de Minas Gerais \\ Departamento de Estatística
}

\begin{abstract}
A técnica de captura-recaptura tem sido utilizada numa variedade muito grande de áreas especialmente na Ecologia e Epidemiologia. Mais recentemente, esta técnica passou a ser utilizada também na indústria para estimar o número total de defeitos (ou falhas) distintos que existem ou podem ser gerados num processo ou produto. Unidades do produto (ou processo) são inspecionadas por um certo número k de inspetores que têm a tarefa de identificar todos os defeitos presentes no produto. Combinando os resultados destas inspecões, estima-se o número total de defeitos distintos que podem ser gerados no produto (ou processo) por meio do uso dos estimadores clássicos de capturarecaptura. Os inspetores fazem o papel das ocasiões de captura. Este modelo permite também que se trate situações nas quais o mesmo conjunto de itens do produto (ou processo) passa por inspeção várias vezes por um mesmo inspetor, ou ainda situações nas quais grupos de inspetores são usados em cada ocasião de captura (inspeção). A proposta deste artigo é apresentar alguns estimadores Bayesianos propostos na literatura dentro do contexto do chamado "problema de estimação de espécies" mostrando que eles são simples de serem utilizados no contexto industrial e fornecem estimativas bastante razoáveis.
\end{abstract}

Palavras-chave: captura-recaptura; inspeção; controle de qualidade; estimadores bayesianos.

Capture-recapture methodology has been used in a variety of fields especially in wildlife and Epidemiological studies. More recently, it started been used also in the industrial context to estimate the total number of distinct defects or failures present in a product or process. Units of the product or the process are inspected by a certain number $k$ of inspectors who have the task to find all the imperfections or defects of the product. Combining the inspections results, an estimate for the total number of distinct defects that can be generated in the product (or process) is calculated using the classical statistical estimators constructed under the capture-recapture principles. The inspectors play the role of the capture occasions. This model also allows to treat situations where the same units of the product (or process) are observed by the same inspector many different times, or when groups of inspectors are used in every capture occasion (inspection). The purpose of this paper is to present some Bayesian estimators proposed in the literature to treat the "species problem estimation", showing that they can be easily used in the industrial context giving reasonable estimate results.

Keywords: capture-recapture; inspection; quality control; bayesian estimators.

\section{Introdução}

A inspeção de produtos na busca de erros ou defeitos é uma preocupação constante tanto para produtos acabados quanto para aqueles em estágios de delineamento, teste ou produção. Basicamente se tem duas situações clássicas: num primeiro momento é necessário ter um mecanismo que permita conhecer os tipos de defeitos (ou falhas) que podem ser gerados pelo processo. A partir deste conhecimento pode-se então, estimar a proporção de ítens nãoconformes gerados pelo processo, de um modo global e de um modo particular, isto é, para cada tipo de defeito isoladamente. Métodos de amostragem sistemática, aleatória simples, ou em vários estágios são frequentemente utilizados para efetuar-se a estimação desta proporção de defeitos sendo a fração amostral de ítens não conformes, o estimador usado para estimar a proporção de ítens não conformes gerados pelo processo. A partir desta estimação se faz o controle rotineiro do processo (Montgomery,1991).

O conhecimento dos tipos diferentes de defeitos que podem surgir no processo de geração de um produto pode ser acessado num primeiro momento, via brainstorm reunindo-se os técnicos que têm 
conhecimento sobre o processo per si e via amostragem do produto quando o processo piloto de produção está em operação, ou já se tem uma unidade do produto delineado (Eick, Loader, et. al., 1993; Porter \& Votta,1998). Neste caso, é comum ter-se operadores (ou inspetores) que têm a tarefa de inspecionar o produto tentando identificar os tipos de defeitos que nele existem. Assim, descobrindo-se a natureza dos defeitos presentes pode-se corrigi-los de modo a melhorar o processo de produção final.

A qualidade deste mecanismo de busca de defeitos depende entre outras coisas, do treinamento dos inspetores e do tempo envolvido na operação de inspeção. Em alguns casos, é comum nesta fase piloto de operação, fazer com que uma mesma unidade do produto, ou uma amostra, seja inspecionada por vários inspetores diferentes tentando assim maximizar o encontro de todos os defeitos. No entanto, por melhor que sejam os inspetores é possível que alguns defeitos existentes no produto inspecionado passem desapercebidos. Além disso, como se está inspecionado apenas uma parte do processo, ou seja apenas as unidades que estão sendo produzidas no presente, não se tem uma segurança total de que defeitos diferentes, daqueles observados na fase piloto, não venham a aparecer nas unidades que serão produzidas no futuro. Portanto, é de interesse que a partir dos defeitos encontrados nas unidades observadas na fase piloto, possa-se estimar o número total de defeitos distintos que poderiam ser observados naquele produto. Deste modo, caso o número de defeitos observados na fase piloto fosse próximo desta estimativa de total, ter-se-ia uma segurança maior de que a melhoria do produto passaria apenas pela correção dos defeitos observados nas unidades inspecionadas. De outro lado, se o número de defeitos distintos observados nas unidades inspecionadas estivesse muito longe desta estimativa de total, ter-seia uma indicação de que novas inspeções seriam necessárias para de fato se alcançar a melhoria final do produto. Estas novas inspeções poderiam ser em novas unidades produzidas, ou mesmo nas unidades previamente inspecionadas com o objetivo de buscar defeitos que passaram desapercebidos pelos inspetores. Estimando-se o total de defeitos distintos do produto tem-se ao mesmo tempo acesso a uma estimativa da efetividade do sistema de inspeção, medida que é utilizada por algumas empresas como um critério para continuidade ou não da inspeção, ou para reinspeção de unidades de produtos (Emam \& Laitenberger,2001).

Portanto, tem-se aqui um problema de estimação no qual a partir do número de defeitos distintos observados nas amostras deseja-se inferir sobre o número de defeitos distintos que estão presentes no processo e que vão aparecer no futuro em outras unidades que serão fabricadas, ou que já estavam presentes nas unidades inspecionadas mas foram perdidos no mecanismo de inspeção. Dentro do contexto estatístico, este problema de estimação é similar ao do chamado "problema da estimação de espécies distintas de uma população", no qual a partir de uma amostra de espécies distintas observadas numa região de estudo, o ecólogo faz a estimação para o número total de espécies distintas que estariam habitando a respectiva região, ou para o número de espécies distintas que estão na região mas não foram observadas na amostra considerada. No contexto ecológico a palavra "espécie" realmente representa algum tipo de animal ou vegetal. No entanto, o termo "espécie" pode ser usado num sentido mais genérico (Mingoti \& Meeden,1992). Na literatura. pode-se chamar de "espécies" as palavras diferentes que um autor usa para escrever um livro, e o uso da amostragem tem o objetivo de estimar o vocabulário do autor (Efron \& Thisted, 1976). Em pesquisa de mercado as "espécies" podem ser as características que o produto precisa possuir para ser atrativo ao consumidor (Mingoti,2001), ou as que um profissional deve ter para ser respeitado no mercado consumidor (Mingoti, 1999). Os números distintos que aparecem numa série numérica finita, ou os elementos que fazem parte de uma população, quando o objetivo é estimar-se o tamanho de alguma população humana ou animal, constituem outros exemplos dentre os muitos que existem na literatura. Dentro do contexto de inspeção de produtos podese considerar cada tipo de defeito como uma "espécie". Defeitos de natureza diferentes são então, tratados como espécies diferentes ou distintas do processo (população). Grande parte dos trabalhos publicados nesta linha referem-se a inspeção de softwares em busca de erros na programação ou codificação, ou mesmo de erros na redação de documentos, manuais, ou procedimentos operacionais. Nestes trabalhos os autores utilizam a técnica estatística conhecida como captura-recaptura (Otis, Burnhan, et. al.,1978) para a estimação do número total de defeitos distintos do produto, uma técnica muito difundida na Ecologia, e que atualmente tem sido muito aplicada na Epidemiologia, ou em estudos de populações humanas (Caiaffa, Mingoti et.al, 2001; Seber, Huakan et. al,. 2000).

\section{Metodologia de captura- recaptura no contexto industrial: breve descrição}

Dentro do contexto industrial o experimento para se obter as informações amostrais necessárias à estimação, via metodologia de captura e recaptura, é conduzido da seguinte forma: primeiramente, escolhese as unidades do produto que participarão do estudo. 
Cada uma destas unidades será então, inspecionada de forma independente por $k$ inspetores selecionados previamente. Presume-se que os inspetores sejam experientes o suficiente para fazer a devida observação das unidades experimentais. Na terminologia de captura-recaptura, os inspetores fazem o papel das ocasiões de captura. A ordem na qual os inspetores farão as inspeções deve ser aleatorizada. Os defeitos encontrados na primeira inspeção, ou seja aquela que é feita pelo inspetor selecionado aletoriamente para iniciar a coleta de informações no experimento, são considerados como os elementos "marcados" na primeira ocasião de captura. Na segunda inspeção, os defeitos encontrados na primeira inspeção poderão ou não ser observados pelo respectivo inspetor. Além disso, pode ser que na segunda inspeção defeitos de natureza diferente daqueles observados na primeira inspeção venham a aparecer. Os defeitos que foram observados na primeira inspeção e voltam a ser observados na segunda inspeção são chamados de "recapturados". Na terceira inspeção podemos ter recapturados de três tipos: aqueles defeitos que apareceram na primeira, segunda e terceira inspeções, aqueles que apareceram na primeira e terceira inspeções ou aqueles que apareceram na segunda e terceira inspeções. O mesmo raciocínio se aplica para as inspeções posteriores. No final do experimento, temos a informação de quantos defeitos distintos apareceram nas $k$ ocasiões de captura (inspeções) e em quais ocasiões eles apareceram. Espera-se de um modo geral, que os defeitos mais comuns sejam captados por quase todos os inspetores, ou seja apareçam em praticamente todas as ocasiões de captura. No entanto, defeitos mais "raros" têm a tendência de serem captados por um número pequeno de inspetores, ou podem mesmo passarem desapercebidos por todos os inspetores participantes do experimento. Entende-se como "raro" um defeito que aparece com baixa frequência, ou ainda um defeito de difícil identificação pelo inspetor.

Dentro desta linha de captura-recaptura modelos probabilisticos são formulados com o intuito de se proceder a estimação. Os modelos mais comuns na área de inspeção de produtos são os chamados: $\mathrm{M}(0)$, $\mathrm{M}(\mathrm{t}), \mathrm{M}(\mathrm{h})$ e $\mathrm{M}(\mathrm{th})$. Cada um desses modelos incorpora uma ou duas fontes de variação (Seber, 1992,1986), como mostrado na Tabela 1 a seguir.

Para maiores detalhes técnicos sobre a construção destes modelos e dos estimadores relacionados sugerimos a leitura de Otis et. al. (1978) e os artigos relacionados com a aplicação na área industrial como: Miller (1999), Briand et. al. (1997) entre outros. É interessante que este modelo de captura-recaptura também se aplica a situações nas quais a inspeção de um mesmo conjunto de unidades do produto (ou processo) é inspecionado várias vezes pelo mesmo inspetor em ocasiões diferentes, e situações nas quais ao invés de se utilizar apenas um inspetor em cada ocasião de inspeção, utiliza-se grupos de inspetores.

Tabela 1. Modelos de Captura e Recaptura e Fontes de Variação

\begin{tabular}{ll}
\hline \hline Modelo & \multicolumn{1}{c}{ Fontes de Variação } \\
\hline $\mathrm{M}(0)$ & $\begin{array}{l}\text { Todos os defeitos têm a mesma probabilidade de } \\
\text { detecção, e todos os inspetores têm a mesma } \\
\text { capacidade de detecção de defeitos. } \\
\text { Todos os defeitos têm a mesma probabilidade de } \\
\text { detecção, mas os inspetores têm capacidades } \\
\text { diferentes de detecção de defeitos. }\end{array}$ \\
$\mathrm{M}(\mathrm{h})$ & $\begin{array}{l}\text { Os defeitos têm probabilidades diferentes de } \\
\text { detecção, mas todos os inspetores têm a mesma } \\
\text { capacidade para detecção de defeitos. }\end{array}$ \\
$\mathrm{M}(\mathrm{th})$ & $\begin{array}{l}\text { Os defeitos tem probabilidades diferentes de } \\
\text { detecção e os inspetores têm capacidades } \\
\text { diferentes de detecção de defeitos. }\end{array}$ \\
\hline
\end{tabular}

Um estimador muito conhecido no caso em que apenas dois inspetores são utilizados no experimento, isto é, temos $k=2$ ocasiões de captura, é o de LincolnPetersen $(1986,1930)$. Sejam $n_{i}, i=1,2$, o número de defeitos encontrados na $i$-ésima inspeção, e $s$ o número de defeitos encontrados simultâneamente na primeira e segunda inspeções, isto é o número de defeitos "recapturados". Então, o estimador de Lincoln-Petersen, construido pelo método estatístico de estimação dos momentos (Garthwaite, Jolliffe $e t$. al, 1995), para o número total de defeitos distintos $(S)$ do produto (ou processo) é definido por:

$$
\hat{S}=\frac{n_{1} n_{2}}{s}
$$

É fácil perceber que quanto maior for o valor de $s$, isto é, dos defeitos "recapturados", menor será o valor de $\hat{S}$. Portanto, quando $s$ é pequeno tem-se a indicação da existência de um grande número de defeitos que estão presentes no processo ou produto, mas que deixaram de aparecer nas duas amostras observadas. Estes são os chamados defeitos "raros". Quando $s=0, \quad$ é infinito. Como um valor $s=0$ tem probabilidade de ocorrência diferente de zero, o estimador de Lincoln-Petersen tem esperança e variância infinitas. Chapman (1951) e Bailey (1951) propuseram uma modificação no estimador (1) com a finalidade de criarem estimadores com média e variância finitas. No entanto, o estimador de LincolnPetersen continua sendo o mais utilizado nos problemas práticos que envolvem apenas duas ocasiões de captura. Para $k>2$, a estimativa de $S$ é obtida através de métodos numéricos que estão implementados por exemplo, no software Capture (Rextad \& Burnham, 1997). 
Alguns estudos publicados na literatura foram desenvolvidos com o objetivo de comparar, dentro do contexto industrial, a qualidade dos vários modelos probabilisticos de estimação usados em capturarecaptura. Em sua grande maioria, estes estudos envolvem processos simulados. O trabalho de Emam \& Laitenberger (1999) é uma excelente referência no caso de duas inspeções. Um outro artigo interessante é o de Briand et. al (1997), um dos poucos que tratam com dados reais, e no qual os autores sabem exatamente qual o número de defeitos distintos envolvidos no processo. Em linhas gerais estes estudos mostram que há uma tendência de subestimar o número total de defeitos distintos e que as estimativas são melhores quando se trabalha com pelo menos 4 inspetores. No entanto, não é aconselhável ter-se experimentos com um número muito elevado de inspetores devido a problemas ou erros que podem advir com a operacionalização da inspeção.

Na maioria dos trabalhos publicados é chamado a atenção para o fato da dificuldade de estimação quando o processo tem muitos defeitos "raros", pois neste caso há sempre uma subestimação de $S$. Daí ser necessário a busca de estimadores que possam ser sensíveis a presença de defeitos "raros" no processo.

\section{Estimadores bayesianos para estimação do total de defeitos distin- tos}

Embora, em grande parte dos trabalhos publicados, os estimadores clássicos relacionados com a aplicação da metodologia de captura-recaptura sejam os utilizados para estimação do número total de defeitos distintos de um produto ou processo, outros estimadores derivados dentro de um contexto mais geral de estimação de espécies distintas de uma população também poderiam ser usados. Dentre os estimadores alternativos, encontram-se os Bayesianos propostos por Mingoti \& Meeden (1992) e Mingoti (1999) e os Não-Paramétricos propostos por Heltshe $\&$ Forrester (1983) e Smith \& Belle (1984). Nosso objetivo não é discutir os estimadores NãoParamétricos mas sim os estimadores Bayesianos propostos por Mingoti (1999) mostrando que estes podem ser facilmente utilizados dentro do contexto industrial. Na apresentação dos estimadores Bayesianos vamos adaptar a notação usada no artigo original para o contexto de estimação de defeitos.

\subsection{Formulação básica dos estimadores bayesianos}

Em Mingoti (1999) um estimador admissivel (Berger,1985), denotado originalmente por , para o verdadeiro valor do número de espécies distintas numa população $S$, foi construido para o caso no qual a amostragem da população em estudo é feita pelo procedimento chamado em Ecologia de "quadrat sampling" ou amostragem por quadrats. Neste esquema a região em estudo é dividida em $N$ subregiões disjuntas, de mesma área, chamadas de quadrats. Destes $N$ quadrats $n, 1<n<N$, são selecionados aleatóriamente para serem observados por algum mecanismo com o objetivo de obter-se a informação das espécies distintas que estão presentes na região amostrada. A dinâmica populacional é descrita em termos da probabilidade $p$ de aparecimento de cada espécie em cada "quadrat" e da distribuição à priori dos valores possíves do número total de espécies distintas na população. Para todas as espécies na população a probabilidade $p$ é assumida como proveniente de uma distribuição Beta de parâmetros . Sabe-se que na distribuição Beta, valores de próximos à zero estão relacionados com distribuições mais concentradas em valores de próximos a zero (Everitt, 1998), isto é, com aquelas populações que apresentam muitas espécies distintas de difícil observação ou captura, que são chamadas de "espécies raras". O termo "rara" de acordo como definido em Mingoti \& Meeden (1992) representa a dificuldade de se observar a espécie, o que pode ser causado pela raridade natural da espécie em termos espaciais ou de abundância, ou pelas limitações operacionais na execução do trabalho de amostragem de campo. No contexto de inspeção de produtos a "espécie rara" seria aquele tipo de defeito que aparece com uma frequência menor quando o produto é manufaturado, ou aquele tipo de defeito que tem uma frequência de aparição não tão pequena mas que por algum motivo passa desapercebido pelos inspetores no processo de inspeção. Isto poderá ocorrer pela dificuldade de reconhecimento do defeito, pelas limitações operacionais do processo de observação dos defeitos, pela falha na definição de que seria um defeito a ser buscado pelo inspetor, ou simplesmente pela negligência dos inspetores. Dentro deste cenário, estima-se o verdadeiro valor de $S$ como sendo:

onde $s$ 'representa o número de espécies distintas observadas na amostra de $n$ quadrats, 


$$
\begin{aligned}
\gamma_{0} & =\int_{0}^{1}(1-p)^{n} \frac{\Gamma(\alpha+\beta)}{\Gamma(\alpha) \Gamma(\beta)} p^{\alpha+1}(1-p)^{\beta-1} d p \\
& =\frac{\Gamma(\alpha+\beta) \Gamma(n+\beta)}{\Gamma(\beta) \Gamma(n+\alpha+\beta)} \quad, 0<\gamma_{0}<1, \alpha>0, \beta>0
\end{aligned}
$$

representa a dificuldade de se observar qualquer espécie da população quando algum procedimento de amostragem é utilizado, e as constantes $(R>0,0<q$ $<$ 1) estão relacionadas com a distribuição Binomial Negativa (Taylor et. al.,1979) que é utilizada como distribuição à priori para o verdadeiro valor de $S$ na construção matemática do estimador $S_{\pi}$. Não vamos aqui repetir as definições apresentadas no artigo original de Mingoti (1999) e nem detalhes técnicos sobre a construção de $S_{\pi}$, mas sim mostrar como os valores de $\left(n, \gamma_{0}, R, q\right)$ seriam interpretados dentro do contexto deste artigo.

Considerando o problema da inspeção de produtos, os inspetores seriam considerados como os "quadrats" amostrais e portanto, teriamos $k=n$ inspetores. A probabilidade $\gamma_{0}$ representaria a dificuldade que cada inspetor teria em observar os diferentes tipos de defeitos presentes na unidade do produto, sendo que valores de $\gamma_{0}$ próximos de 1 , ou equivalentemente valores de $\alpha$ próximos de zero, estariam indicando que haveria um grande número de defeitos distintos que seriam difícies de serem observados ou identificados numa inspeção, e que muito provavelmente tenderiam a não aparecer nas $n$ inspeções consideradas no experimento. Para situações como estas, o valor observado de defeitos distintos na amostra , tende a ser muito menor que o valor verdadeiro de $S$. O parâmetro $q$ estaria representando a probabilidade à priori de que os defeitos distintos fossem observados por qualquer inspetor do experimento, ou de outro modo, a proporção de defeitos distintos presentes no produto (ou processo). Finalmente, o parâmetro $R$ estaria relacionado com a frequência de observação de cada tipo de defeito entre os vários inspetores, ou em outras palavras, seria um índice de agregação de defeitos ao longo do processo (produto). Altos valores de $R$ indicam que os defeitos distintos apareceriam de uma forma mais diepersa no processo (produto), enquanto que baixos valores de $R$ indicam que os defeitos apareceriam em forma de conglomerados.

Pode ser facilmente notado que para valores fixos de $R$ e $q$ o estimador $S_{\pi}$ é uma função crescente de $\gamma_{0}$ e quando $\gamma_{0} \rightarrow 1$ este estimador converge para:

$$
S_{\pi}^{*}=s^{\prime}+\frac{\left(R+s^{\prime}\right) q}{(1-q)}=\frac{s^{\prime}+R q}{(1-q)}
$$

Um terceiro estimador apresentado em Mingoti (1999) e chamado de $S_{\pi *}$ definido por:

$$
S_{\pi^{*}}=\frac{s^{\prime}+\gamma_{0}}{\left(1-\gamma_{0}\right)}
$$

é um caso particular de $S_{\pi}$ quando $R=1$ e $q$ converge para 1 enquanto $\gamma_{0}$ permance fixo.

Um aspecto interessante dos estimadores $S_{\pi}, S_{\pi}^{*}$ e $S_{\pi^{*}}$ é que eles não dependem do valor inicial de divisão $N$, que poderia constituir num limitante para seu uso no caso da inspeção de produtos. Além disso, $S_{\pi}$ e $S_{\pi}^{*}$ são estimadores superiormente limitados para $0 \leq q<1$.

Na construção dos estimadores $\quad, S_{\pi}^{*}$ e $S_{\pi^{*}}$ os parâmetros $(\alpha, \quad, R, q)$ são assumidos como conhecidos pelo pesquisador o que na prática nem sempre é possível. No entanto, Mingoti \& Meeden (1992) mostraram que os parâmetros ( , ) da distribuição Beta podem ser estimados por máxima verossimilhança (Garthwaite, Jolliffe et. al. ,1995). Este procedimento de estimação basicamente considera a função de densidade condicional do vetor

$$
\text { dado } s^{\prime}=\sum_{i=1}^{n} D_{i} \text {, onde } D_{i}
$$

representa o número de defeitos observados por exatamente $i$ inspetores, $i=1,2, \ldots n$. Esta função de densidade depende dos parâmetros ( $\alpha, \quad)$. Estimando-se estes parâmetros tem-se automaticamente uma estimativa da probabilidade de acordo com a expressão (3). De acordo com Mingoti (1999) os parâmetros $(R, q)$ podem ser estimados utilizando-se as quantidades amostrais $R_{s}=\left[n / s^{\prime}\right], q_{s}=D / s^{\prime}$, respectivamente, onde $D$ representa o número de defeitos distintos observados exatamente por um dos inspetores, ou seja o número de defeitos distintos "capturados" uma única vez no processo de inspeção, e [w] denota o maior inteiro menor ou igual a w, sendo $R_{S}$ definido como 1 quando $\mathbf{w}$ for menor que 1. Quando o valor de $q_{s}$ é grande tem-se a indicação de que há um grande número de defeitos raros no processo, uma vez que $q_{s}$ depende do valor de $D$ que está relacionado com o número de defeitos raros no produto. Deste modo, quando $D$ é grande tem-se uma indicação de que o processo gera defeitos raros com uma grande frequência, ou em outras palavras esperase que muitos dos defeitos existentes no processo não sejam observados ou identificados no momento das inspeções.

A qualidade dos estimadores $S_{\pi}, S_{\pi}^{*}$ e $S_{\pi^{*}}$ já foi objeto de discussão nos artigos de Mingoti (1999) e Mingoti (2001). Nestes artigos mostrou-se que estes 
estimadores apresentam um bom desempenho em problemas práticos e são particularmente úteis em situações nas quais a população tem um grande número de "espécies" raras que não são observadas na amostra, fato este que contribui para que outros estimadores clássicos não tenham uma boa qualidade pois nestes casos, tendem a subestimar o verdadeiro número de espécies distintas da população.

Considerando-se que uma das grandes preocupações apresentadas nos artigos que utilizam a metodologia de captura e recaptura para estimação do número total de defeitos distintos do processo (ou produto) é exatamente a subestimação de $S$ quando o processo ou tem defeitos raros ou o mecanismo de inspeção não é muito confiável, os estimadores $S_{\pi}$, $S_{\pi}^{*}$ e $S_{\pi^{*}}$ podem ser vistos como alternativos devido a sensibilidade que têm em relação a existência de "espécies" raras no processo. A seguir apresentamos um exemplo de aplicação.

\subsection{Exemplo de aplicação}

Um manual de operações foi inspecionado por 6 inspetores independentemente. No final da inspeção foram observados 24 defeitos diferentes no documento sendo que 10 deles apareceram apenas uma vez, ou seja foram detectados apenas por um dos inspetores. Neste caso, tem-se $n=6, s^{\prime}=24, D=10$, $q_{s}=\frac{10}{24}=0,4166, R_{s}=\frac{6}{24}<1 \Rightarrow R_{s}=1$, e usando-se o processo de estimação descrito em Mingoti \& Meeden (1992) para estimar $\gamma_{0}$ chega-se a estimativa: $\hat{\gamma}_{0}=0,3502$. Portanto, teriamos as seguintes estimativas Bayesianas para o valor verdadeiro do número de defeitos distintos do manual inspecionado:

$$
S_{\pi}=\frac{s^{\prime}+R q \gamma_{0}}{\left(1-q \gamma_{0}\right)}=\frac{24+(0,4166)(0,3502)}{(1-(0,4166)(0,3502))}=28,27 \approx 29
$$

defeitos

(2) $S_{\pi}{ }^{*}=\frac{s^{\prime}+R q}{(1-q)}=\frac{24+0,4166}{(1-0,4166)}=41,85 \approx 42$

defeitos

(3) $S_{\pi^{*}}=\frac{s^{\prime}+\gamma_{0}}{\left(1-\gamma_{0}\right)}=\frac{24+0,3502}{(1-0,3502)}=37,47 \approx 38$

defeitos

Os resultados dos três estimadores são diferentes pois refletem realidades diferentes. Os estimadores $S_{\pi}^{*}$ e $S_{\pi^{*}}$ são formas limites de $S_{\pi}$. No primeiro caso $\left(S_{\pi}^{*}\right)$, o pesquisador estaria assumindo à priori que o número de defeitos que poderiam passar desapercebidos no processo de inspeção é alto, uma vez este estimador é construido considerando-se $\gamma_{0} \rightarrow 1$. No segundo caso ( $S_{\pi^{*}}$ ), o pesquisador estaria assumindo à priori que o número de defeitos distintos do processo é alto uma vez que este estimador é construido considerando-se $q \rightarrow 1$. O estimador por outro lado, não incorpora nenhuma destas suposições à priori, e permite que os dados amostrais estimem tanto a probabilidade $\gamma_{0}$ quanto a probabilidade $q$. Ao nosso ver este seria o estimador mais adequado nas aplicações práticas de um modo geral.

\section{Análise de qualidade via simula- ção}

Nesta seção vamos apresentar os resultados de um pequeno estudo de simulação feito para avaliar a qualidade dos estimadores $S_{\pi}, S_{\pi}^{*}$ e $S_{\pi^{*}}$ em situações nas quais o número verdadeiro de $S$ se aproxima daqueles encontrados em situações de inspeções industriais. Embora, resultados de estudos simulados ou de populações reais tenham sido apresentados em Mingoti $(1999,2001)$, o valor de $S$ naqueles casos são muito grandes comparados àqueles que podem aparecer no contexto de inspeção de defeitos.

Para esta nova simulação os diferentes tipos de defeitos e a frequência dos mesmos foram gerados através de um processo estocástico $\left\{Y_{t}, t \in \mathfrak{R}\right\}$ Auto Regressivo de ordem 1 (Brockwell \& Davies,1991), com parâmetro $\phi$ no conjunto $\{0,0$; $0,2 ; 0,5 ; 0,9\}$. Para cada valor de foram geradas 200 séries de tamanho 250 mantendo-se os erros aleatórios do modelo provenientes de uma distribuição Normal com média zero e variância $=4$. Os valores gerados em cada série foram arredondados para o inteiro mais próximo. Deste modo, cada número inteiro distinto que apareceu na série foi considerado como um defeito de natureza distinta. Após este procedimento, a série gerada foi dividida em 25 subconjuntos de mesmo tamanho preservandose a ordem em que os números foram gerados dentro da série. Cada um destes subconjuntos equivaleria a uma unidade de produto. Portanto, temos uma população com 25 unidades de produto. Amostras aleatórias de tamanho $n=3,5,8,12,15,18$ sem reposição foram então, selecionadas dentre estas 25 unidades de produto para inspeção. Supondo-se que cada unidade de produto na amostra é inspecionda por um inspetor diferente, estariamos próximos da situação na qual temos $n$ inspetores e cada inspetor avalia uma unidade diferente do produto (ou processo) em estudo. Para cada série o número total de defeitos 
Tabela 2. Resultados da simulação considerando associação positiva entre inspeções

\begin{tabular}{|c|c|c|c|c|c|c|c|c|}
\hline \multicolumn{9}{|c|}{$(\phi=0,00 ; \quad S=23)$} \\
\hline \multirow[t]{2}{*}{$n$} & \multicolumn{2}{|c|}{ Estimador $S_{\pi}$} & \multicolumn{2}{|c|}{ Estimador $S_{\pi}^{*}$} & \multicolumn{2}{|c|}{ Estimador $_{\pi^{*}}$} & \multicolumn{2}{|c|}{ Valores Médios } \\
\hline & $E M$ & $R E Q M$ & $E M$ & $R E Q M$ & $E M$ & $R E Q M$ & $\hat{\gamma}_{0}$ & $d p \hat{\gamma}_{0}$ \\
\hline 3 & $-4,8618$ & 5,2860 & 2,2380 & 5,3850 & $-1,5600$ & 4,2600 & 0,1919 & 0,0982 \\
\hline 5 & $-3,8824$ & 4,1745 & 0,7026 & 3,4301 & $-1,5800$ & 3,3100 & 0,1211 & 0,0710 \\
\hline 8 & $-2,5910$ & 2,9383 & 1,2306 & 3,2271 & $-0,6190$ & 2,4520 & 0,0965 & 0,0491 \\
\hline 12 & $-1,5100$ & 1.8565 & 1,9548 & 3,0652 & 0,3290 & 1,7510 & 0,0861 & 0,0303 \\
\hline 15 & $-1,0538$ & 1,4458 & 1,9682 & 2,9085 & 0,6010 & 1,5810 & 0,0755 & 0,0214 \\
\hline 18 & $-0,5046$ & 0,8810 & 2,3512 & 3,0223 & 1,1088 & 1,5152 & 0,0719 & 0,0145 \\
\hline \multirow{3}{*}{$n$} & \multirow{2}{*}{\multicolumn{2}{|c|}{ Estimador $S_{\pi}$}} & \multicolumn{2}{|r|}{$(\phi=0,20$} & \multicolumn{2}{|l|}{$S=27)$} & & \\
\hline & & & \multicolumn{2}{|c|}{ Estimador $S_{\pi}^{*}$} & \multicolumn{2}{|c|}{ Estimador $S_{\pi^{*}}$} & \multicolumn{2}{|c|}{ Valores Médios } \\
\hline & $E M$ & $R E Q M$ & $E M$ & $R E Q M$ & $E M$ & $R E Q M$ & $\hat{\gamma}_{0}$ & $d p \hat{\gamma}_{O}$ \\
\hline 3 & $-7,6328$ & 8,1340 & 0,6260 & 6,5680 & $-3,1700$ & 6,2600 & 0,2145 & 0,1051 \\
\hline 5 & $-5,9397$ & 6,3914 & 0,1894 & 5,1537 & $-2,3700$ & 5,0700 & 0,1690 & 0,0918 \\
\hline 8 & $-4,2566$ & 4,7395 & 0,9620 & 4,3897 & $-0,9380$ & 3,7890 & 0,1448 & 0,0650 \\
\hline 12 & $-2,6041$ & 3,0470 & 2,6877 & 4,1722 & 0,7630 & 2,7810 & 0,1392 & 0,0418 \\
\hline 15 & $-1,66,13$ & 2,2807 & 3,7126 & 4,9154 & 1,6400 & 3,0750 & 0,1324 & 0,0375 \\
\hline 18 & $-1,0468$ & 1,6261 & 4,1266 & 4,8284 & 2,1199 & 2,9159 & 0,1243 & 0,0163 \\
\hline \multirow{3}{*}{$n$} & \multirow{2}{*}{\multicolumn{2}{|c|}{ Estimador $S_{\pi}$}} & \multicolumn{4}{|c|}{$(\phi=0,50 ; \quad S=26)$} & & \\
\hline & & & \multicolumn{2}{|c|}{ Estimador $S_{\pi}^{*}$} & \multicolumn{2}{|c|}{ Estimador $S_{\pi^{*}}$} & \multicolumn{2}{|c|}{ Valores Médios } \\
\hline & $E M$ & $R E Q M$ & $E M$ & $R E Q M$ & $E M$ & $R E Q M$ & $\hat{\gamma}_{0}$ & $d_{p} \hat{\gamma}_{O}$ \\
\hline 3 & $-5,5411$ & 6,4820 & 3,7070 & 8,1010 & $-1,3400$ & 5,9500 & 0,2198 & 0,1075 \\
\hline 5 & $-4,4803$ & 5,0347 & 1,6736 & 5,0042 & $-1,5400$ & 4,3700 & 0,1396 & 0,0831 \\
\hline 8 & 2,6402 & 3,1246 & 2,8357 & 4,5492 & 0,2650 & 2,8410 & 0,1281 & 0,0555 \\
\hline \multirow{2}{*}{$\frac{12}{15} 1$} & $-1,2346$ & 1,9260 & 3,0238 & 4,3392 & 1,4710 & 2,8040 & 0,1094 & 0,0384 \\
\hline & $-0,6068$ & 1,2171 & 2,9417 & 3,7974 & 1,8500 & 2,4610 & 0,0962 & 0,0244 \\
\hline 18 & $-0,2213$ & 0,7632 & 2,7419 & 3,2483 & 2,0252 & 2,2953 & 0,0858 & 0,0163 \\
\hline \multirow{3}{*}{$n$} & \multicolumn{6}{|c|}{$(\phi=0,90 ; \quad S=41)$} & & \\
\hline & \multicolumn{2}{|c|}{ Estimador $S_{\pi}$} & \multicolumn{2}{|c|}{ Estimador $S_{\pi}^{*}$} & \multicolumn{2}{|c|}{ Estimador $S_{\pi^{*}}$} & \multicolumn{2}{|c|}{ Valores Médios } \\
\hline & $E M$ & $R E Q M$ & $E M$ & $R E Q M$ & $E M$ & $R E Q M$ & & $d p^{\gamma_{0}}$ \\
\hline 3 & $-8,1455$ & 9,6710 & 13,0880 & 17,2440 & 1,1000 & 9,5300 & 0,3315 & 0,1088 \\
\hline 5 & $-6,7369$ & 7,4725 & 5,9060 & 8,8537 & 0,2900 & 6,2600 & 0,2179 & 0,0834 \\
\hline 8 & $-4,4009$ & 5,1859 & 4,4658 & 7,0558 & 1,3640 & 5,4510 & 0,1593 & 0,0681 \\
\hline 12 & $-2,9257$ & 3,4989 & 3,0710 & 4,7468 & 1,3810 & 3,9200 & 0,1121 & 0,0470 \\
\hline 15 & $-2,0281$ & 2,5587 & 3,3505 & 4,5708 & 1,6260 & 3,2750 & 0,0937 & 0,0351 \\
\hline 18 & $-1,0860$ & 1,6391 & 4,1611 & 4,7900 & 2,4240 & 3,2168 & 0,0885 & 0,0243 \\
\hline
\end{tabular}

distintos $S$ é uma variável aleatória e seu valor somente é conhecido após a geração de todos os valores da série. A forma utilizada para geração dos dados cria uma certa estrutura de associação entre os números inteiros que representam os defeitos, pois num processo Auto-Regressivo de ordem 1 a correlação entre os valores da série é representada pelo parâmetro sendo a correlação entre unidades espaçadas em h unidades dada por:

Portanto, cria-se uma associação entre os tipos de defeitos que aparecem em cada um dos 25 subconjuntos, chamados aqui de unidades do produ- to. Em nossa simulação o caso em que $\phi=0$ corresponde à situação na qual não há associação e os tipos de defeitos aparecem aleatoriamente. $\mathrm{O}$ caso em que $\phi=0,90$ representa a maior associação investigada. $\mathrm{Na}$ inspeção de produtos é natural pensarmos que exista algum grau de associação entre as unidades de produto que serão avaliadas pelos inspetores e, portanto deve haver algum tipo de associação entre os resultados que serão observados nas várias inspeções realizadas. Para cada série gerada de acordo com o valor do parâmetro $\phi$, calculou-se os valores dos estimadores $\quad, S_{\pi}^{*}$ e $S_{\pi^{*}}$ sendo estes valores comparados com o valor real $S$ da respectiva série. Assim obtivemos os valores do Erro Médio (EM) e Raiz Quadrada do Erro Quadratico Médio (REQM) de cada um dos estimadores. Os 
resultados obtidos encontram-se na Tabela 2. Como pode ser notado no caso em que não há associação entre defeitos $(\phi=0,0)$, os valores de $E M$ e $R E Q M$ são bastante razoáveis e ficam menores à medida que o número de inspetores (ou ocasiões de captura) aumentam, o que é naturalmente esperado. A partir de $n=8$ os resultados foram muito bons para os três estimadores comparados. Nesta faixa, a probabilidade estimada está no intervalo de [0,0719; 0,095], indicando que a perda esperada de defeitos no processo de inspeção é pequena. Quando um nível de associação não muito acentuado é introduzido entre inspeções $(\phi=0,20)$, o mesmo comportamento geral é observado nos valores de $E M$ e $R E Q M$ sendo que os melhores resultados ocorrem a partir de $n=12$. Neste caso, a probabilidade estimada aumenta em relação ao caso $(\phi=0,0)$ e para está na faixa de $[0,1243 ; 0,1392]$. Para $=0,50$, os melhores resultados ocorrem para e para $=0,90$ os melhores resultados ocorrem a partir de $n$ igual a 12 . É interessante observar que o estimador tem um comportamento ruim quando $\phi=0,90$ pois superestima demasiadamente o verdadeiro valor de $S$. Isto ocorre devido ao fato de estimada ter um valor alto (0,3315). Dado ao mecanismo de construção do estimador Bayesiano, valores mais altos de $\gamma_{0}$ indicam a existência de muitas espécies "raras" (defeitos "raros") daí a superestimação de $S$ quando $\phi=0,90$. Ressalta-se o bom desempenho do estimador em todas as análises.

\section{Considerações finais}

Neste artigo procuramos mostrar que o problema de estimação do número total de defeitos distintos de um produto ou processo, pode ser visto como um caso particular do tradicional "problema de estimação do número total de espécies distintas de uma população". Ao vermos o problema desta forma, podemos utilizar estimadores alternativos aos clássicos de captura-recaptura, que são tradicionalmente usados para estimar o tamanho populacional. Mostramos que os estimadores Bayesianos propostos por Mingoti $(1999,2001)$ são alternativas interessantes e que se aplicam para o problema de estimação no contexto da inspeção de produtos. Estes estimadores podem ser adaptados à situações diversas de comportamento entre a geração de defeitos do produto (ou processo) ou entre inspetores uma vez que dependem do parâmetro $\gamma_{0}$ que controla a esperança do pesquisador em encontrar ou não defeitos "raros" no produto (ou processo) avaliado. Os resultados da simulação foram bastante satisfatórios indicando que os estimadores Bayesianos de um modo geral tiveram boa qualidade.

\section{Referências bibliográficas}

BRIAND, L. C.; EMAN, K.E.; FREIMUT, B.; LAITENBERGER, O. Quantitative evaluation of capture-recapture models to control software inspection, $8^{\text {th }}$ International Symposium on Software Reliability Engineeering (ISSRE's 97), Alburquerque, NM, 234-244,1997.

BROCKWELL,P.J.; DAVIES,R.A. Time Series Theory and Methods. New York: Springer Verlag, 1991, 519 p.

CAIAFFA, W.; MINGOTI, S.A.; PROIETTI, F.; CARNEIRO-PROIETTI, A. B.;MARQUES, L.; MENDONÇA, E. A.; DESLANDES, S.; DONEDA, D.; SILVA, R.C.; EQUIPE PROJETO AJUDE

BRASIL. Projeto Ajude-Brasil: Avaliação Epidemiológica dos Usuários de Drogas Injetáveis dos Projetos de Redução de Danos (PRD) Apoiados pela $C N-D S T / A I D S$. Brasília: Ministério da Saúde, Brasil, 2001, 341 p.

BAILEY, N.T.J. On estimating the size of mobile populations from capture-recapture data. Biometrika, 38, 293-306, 1951.

BERGER, J. O. Statistical Decision Theory and Bayesian Analysis. 2a. Edição, New York: Springer Verlag, 1985, 616 p.

CHAPMAN, D. G. Some properties of the hypergeometric distribution with applications to zoological censuses. University of California Publications Statistics, 1, 131-160, 1951.

EFRON, B. \& THISTED, R. Estimating the number of unseen species: how many words did Shakespeare know? Biometrika, 63, 435-447, 1976.

EICK, S. G..; LOADER, C. R.; LONG, M. D.; VOTTA, L. G..; WIEL, S. V. Estimating software fault content before coding, Proceedings of the $15^{\text {th }}$ International Conference of Software Engineering, Melbourne, 59-65, 1993.

EMAM, K. E.; LAITENBERGER, O. Evaluating capture-recapture models with two inspectors. IEEE Trans-Software Engineering, 27,9,851-864,2001.

EVERITT, B.S. The Cambridge Dictionary of Statistics. Cambridge: Cambridge University Press, 1998, 360 p.

GARTHWAITE,P.H.; JOLLIFFE, I.T.; JONES, B. Statistical Inference. New York: Prentice Hall, 1995, 290 p. 
HELTSHE, J. F.; FORRESTER, N. E. Estimating species richness using jackknife procedure, Biometrics, 39, 1-12, 1983.

LINCOLN, F. C. Calculating waterfowl abundance on the basis of banding returns, U.S. Department of Agricultural Circular, 118, 1-4, 1930.

MILLER, J. Estimating the number of remaining defects after inspection, Software Testing, Verification \& Reliability, 9, 3, 167-189,1999.

MINGOTI, S. A. How to estimate the amount of important characteristics missing in a consumers sample by using bayesian estimators. Pesquisa Operacional, 21,1, 31-38, 2001.

MINGOTI, S. A. Bayesian estimator for the total number of distinct species when quadrat sampling is used, Journal of Applied Statistics, 26, 4, 469$483,1999$.

MINGOTI, S. A. \& MEEDEN, G. Estimating the total number of distinct species using presence and absence data, Biometrics, 48, 863-875, 1992.

MONTGOMERY, D.C. Introduction to Statistical Quality Control . New York: John Wiley, 1991, 752 p.

OTIS, D., BURNHAN, K.P.; WHITE, G.C. \& ANDERSON, D.R. Statistical inference from capture data on closed populations, Wildlife Monographs, 62, 1-135, 1978.

PORTER, A., VOTTA, L. Comparing detection methods for software requirements inspections: a replication using professional subjects, Empirical Software Engineering: An International Journal, 3, 4, 335-379, 1998.

PETERSEN, G.G.J. The yearly immigration of young plaice into the limfjord from the german sea, Report of Danish Biology Statistics, 6, 1-48., 1896.

REXTAD, E., BURNHAN, K. User's guide for interative program Capture. Alaska,1997.

SEBER, G. A. F. A review of estimating animal abundance, Biometrics, 42, 267-292, 1986.

SEBER, G. A. F. A review of estimating animal abundance II, International Statistical Review, 60, 2, 129-166, 1992.

SEBER, G. A. F.; HUAKAU, J. T.; SIMMONS, D. Capture-Recapture: Epidemiology, and list mismatches: two lists, Biometrics, 56, 1227-1232, 2000.
SMITH, L. R. \& BELLE, G. V. Nonparametric estimation of species richness, Biometrics, 40, 119129, 1984.

TAYLOR, L.R.; WOIWOD, I.P.; PERRY, J.N. The negative binomial as a dynamic ecological model for aggregation, and the density dependence of $k$, Journal of Animal Ecology, 48, pp. 289-304, 1979. 